amylase would have been the sole reason for imaging/hospital referral. At a cost of $£ 45$ per test, it may be financially and clinically helpful to have decision support tools for primary care physicians' requests.

\section{PWE-075 BILIARY BRUSH CYTOLOGY: IS IT WORTH PURSUING? A SINGLE CENTRE EXPERIENCE}

Emmanuel Selvaraj ${ }^{\star}$, Ian Lei, Aniko Rendek, Elena Collantes, Caroline Hughes, Adam Bailey. Oxford University Hospitals NHS Foundation Trust, London, UK

10.1136/gutjnl-2019-BSGAbstracts.306

Introduction The diagnostic sensitivity of endoscopic retrograde cholangiopancreatography (ERCP) brush cytology is typically variable (6-64\%) and reported to be precisely $41.6 \%$ in a recent meta-analysis ${ }^{1}$. The perceived futility coupled with the emergence of endoscopic ultrasound (EUS) sampling with a higher reported sensitivity (74\%), has curbed the enthusiasm to obtain brush cytology in suspected pancreaticobiliary malignancies. This study aims to investigate the diagnostic yield from biliary brush cytology following introduction of changes to practice in 2014 at a single centre tertiary hospital.

Methods All adult patients who had an index brush cytology for biliary stricture at ERCP from January 2015 to January 2018 were identified and included in this retrospective study. Electronic patient records were searched for corresponding histocytopathology data, radiology report, multidisciplinary team (MDT) meeting outcome and clinic letters. Cytological diagnosis was compared with the final outcome of malignant or benign. Patients were considered to have a malignant outcome if (i) subsequent tissue sampling or resection confirmed primary or metastatic disease or (ii) there was unequivocal radiological or clinical progression of malignant disease. Patients were considered to have a benign outcome if (i) subsequent resection was benign or (ii) clinical follow-up for a minimum of 12 months was uneventful. Sensitivity, specificity, positive predictive value (PPV), negative predictive value (NPV) and accuracy were calculated. Differences in yield between cancers were assessed using Chi-square and Fisher's exact test

Results 127 patients were included with a mean age at presentation of 69.5 years $(62 \%$ male). Cytological diagnosis were as follows: malignant $50.4 \%$, suspicious $14.2 \%$, atypical $11.0 \%$, negative $23.6 \%$ and non-diagnostic $0.8 \%$. $79.5 \%$ of strictures had a malignant outcome with $53.5 \%$ pancreatic cancer, $38.6 \%$ cholangiocarcinoma, $3.0 \%$ ampullary cancer and $4.9 \%$ other. Diagnostic performance test characteristics are as follows: sensitivity 79.2\%, specificity 96.2\%, PPV 98.8\%, NPV $54.3 \%$ and accuracy $82.7 \%$. There was no statistically significant difference in yield between pancreatic cancer and cholangiocarcinoma $(p=0.80)$. Workforce restructuring and changes to laboratory practice in histopathology department significantly contributed to our higher yield.

Conclusion Biliary brush cytology yield in our centre is higher than reported in the literature and remains a valuable tool in the diagnosis of pancreaticobiliary malignancies. Multifaceted changes in practice can produce comparable yield to EUS sampling.

\section{REFERENCE}

1. Burnett AS, Bailey J, Oliver JB, et al. Sensitivity of alternative testing for pancreaticobiliary cancer: a 10-y review of the literature. J Surg Res 2014;190:535-47.

\section{Small Bowel}

Orals

\section{OWE-16 DEVELOPMENT AND CLINICAL VALIDATION OF A GENETIC RISK SCORE FOR COELIAC DISEASE}

${ }^{1}$ Seth Sharp*, 'Samuel Jones, ${ }^{4}$ Robert Kimmitt, ${ }^{1}$ Michael Weedon, ${ }^{2}$ Anne Halpin,

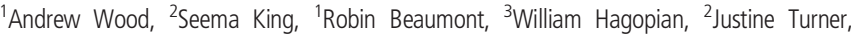
${ }^{1,4}$ Richard Oram. 'University of Exeter Medical School, Exeter, UK; ${ }^{2}$ University of Alberta, Edmonton, Canada; ${ }^{3}$ Pacific Northwest Research Institute, Seattle, USA; ${ }^{4}$ Royal Devon and Exeter NHS Foundation, Exeter, UK

\subsection{6/gutjnl-2019-BSGAbstracts.307}

Introduction Specific HLA-DQ genes predispose to coeliac disease (CD) and HLA typing is occasionally used as a rule-out test in clinic. However, $\mathrm{CD}$ is polygenic and genome wide association studies (GWAS) have implicated $\sim 40$ additional genetic variants. Using single nucleotide polymorphisms (SNPs) we aimed to combine all associated variants into a genetic risk score and assess its utility as a clinical tool.

Methods We used imputation to identify SNPs strongly correlated $\left(\mathrm{r}^{2}>0.95\right)$ with 4 key HLA-DQ haplotypes (DQ2.5/ DQ2.2/DQ7.5/DQ8) in UK Biobank. We derived HLA-DQ odds ratios from 12,041 cases and 12,228 controls (Wellcome Trust). We combined this with additional SNPs from recent GWAS to generate a coeliac genetic risk score (C-GRS).

We validated the C-GRS in a population based cohort (UK Biobank) with 1237 cases identified by hospital admission codes. We genotyped the C-GRS in 161 samples from a paediatric clinic where patients had been assessed using antitissue transglutaminase antibodies, biopsy and HLA typing.

Results The C-GRS consisted of 42 SNPs and was highly discriminative of CD in UKBiobank. The C-GRS was more discriminative than HLA stratification alone (ROC-AUC $=0.88$ [95\%CIs:0.87-0.89] v 0.81, p<0.0001) and highly discriminative in the paediatric clinic (ROC-AUC $=0.82$ [95\%CIs:0.750.90], $\mathrm{p}<0.0001)$.

Conclusions A C-GRS can aid in identifying incident cases of $\mathrm{CD}$ and is more effective than HLA typing alone. Given the low costs of SNP genotyping relative to HLA typing a C-GRS could improve the availability and utility of coeliac genetic testing in $\mathrm{CD}$ diagnosis and in recruitment to research studies.

\section{OWE-18 NON-RESPONSIVE AND REFRACTORY COELIAC DISEASE: THE LARGEST UK EXPERIENCE FROM THE NHS ENGLAND NATIONAL CENTRE}

${ }^{1}$ Elisabeth MR Baggus*, ${ }^{1}$ Anupam Rej, ${ }^{1,2}$ Annalisa Schiepatti, ${ }^{1}$ Lauren J Marks, ${ }^{1}$ Nick Trott, ${ }^{1}$ Marios Hadjivassiliou, 'David S Sanders. 'Academic Unit of Gastroenterology, Royal Hallamshire Hospital, University of Sheffield, Sheffield, UK; ${ }^{2}$ Coeliac Centre, University of Pavia, Italy

\subsection{6/gutjnl-2019-BSGAbstracts.308}

Introduction Non-responsive coeliac disease (NRCD) is defined by persisting symptoms or laboratory abnormalities in patients with coeliac disease (CD) despite a gluten-free diet (GFD). Causes of NRCD are heterogeneous, with refractory CD (RCD) being associated with poor prognosis. The aims of this study are to identify the aetiologies for persisting symptoms in patients with NRCD referred to a national UK centre for CD, and to assess mortality rates in each group. 